\title{
A Complex Solar Coronal Jet with Two Phases
}

\author{
Jie Chen ${ }^{1,2}$, Jiangtao $\mathrm{Su}^{1,3}$, Yuanyong Deng ${ }^{1}$, and E. R. Priest ${ }^{4}$ \\ ${ }^{1}$ Key Laboratory of Solar Activity, National Astronomical Observatories, Chinese Academy of Sciences, Beijing 100012, China; chenjie@bao.ac.cn \\ ${ }^{2}$ State Key Laboratory for Space Weather, Center for Space Science and Applied Research, Chinese Academy of Sciences, Beijing 100190, China \\ ${ }^{3}$ School of Astronomy and Space Sciences, University of Chinese Academy of Sciences, 19A Yuquan Road, Beijing 100049, China \\ ${ }^{4}$ Mathematical Institute, University of St Andrews, North Haugh, St Andrews, KY16 9SS, UK \\ Received 2016 November 5; revised 2017 April 4; accepted 2017 April 6; published 2017 May 4
}

\begin{abstract}
Jets often occur repeatedly from almost the same location. In this paper, a complex solar jet was observed with two phases to the west of NOAA AR 11513 on 2012 July 2. If it had been observed at only moderate resolution, the two phases and their points of origin would have been regarded as identical. However, at high resolution we find that the two phases merge into one another and the accompanying footpoint brightenings occur at different locations. The phases originate from different magnetic patches rather than being one phase originating from the same patch. Photospheric line of sight (LOS) magnetograms show that the bases of the two phases lie in two different patches of magnetic flux that decrease in size during the occurrence of the two phases. Based on these observations, we suggest that the driving mechanism of the two successive phases is magnetic cancellation of two separate magnetic fragments with an opposite-polarity fragment between them.
\end{abstract}

Key words: magnetic reconnection - magnetohydrodynamics (MHD) - Sun: activity - Sun: corona Sun: magnetic fields

Supporting material: animations

\section{Introduction}

Jets are transient impulsive phenomena with plasma motions along particular directions (Shibata et al. 1992, 1994; Canfield et al. 1996; Moore et al. 2010; Sterling et al. 2015). They are ubiquitous in the solar atmosphere and occur in active regions, the quiet Sun, and polar regions (Cirtain et al. 2007; Shen et al. 2012; Liu et al. 2014; Zhang \& Ji 2014; Hong et al. 2016; Raouafi et al. 2016; Zhang et al. 2016). The typical length of jets is $10-400 \mathrm{Mm}$, the width is $5-100 \mathrm{Mm}$, and the apparent velocity is $10-1000 \mathrm{~km} \mathrm{~s}^{-1}$ with an average value of $200 \mathrm{~km} \mathrm{~s}^{-1}$ (Shimojo et al. 1996, 1998).

It is generally believed that solar jets are caused by magnetic reconnection. However, the jet characteristics largely depend on the magnetic environment at the jet source. Heyvaerts et al. (1977), Forbes \& Priest (1984), Shibata et al. (1992), and Moore et al. (2013) presented a standard model for the formation of a jet. The essential idea of this model is that at the base of a jet during the emergence of a magnetic arch into an ambient unipolar field, a current sheet forms between the ambient field and one leg of the arch with opposite polarity. During the emergence, the current sheet becomes larger and thinner, until magnetic reconnection is initiated. Interchange reconnection between closed field and open field occurs at the current sheet. The hot plasma on the open field lines escapes out to become the spire of the jet, and the hot plasma on the closed field lines is seen as a bright point at the jet base. This model has been developed further using 2D numerical experiments by Yokoyama \& Shibata $(1995,1996)$, Nishizuka et al. (2008), and Jiang et al. (2012) and, more recently, 3D experiments by Moreno-Insertis \& Galsgaard (2013), NóbregaSiverio et al. (2016), Archontis \& Hood (2013), Syntelis et al. (2015), and Raouafi et al. (2016).

Jets are associated with both flux emergence and cancellation (Chae et al. 1999; Brooks et al. 2007). After new flux has emerged, one or another flux element often cancels with a nearby one of opposite polarity. Most of them occur in mixed polarity regions (Shimojo et al. 1996). Jets are commonly associated with magnetic cancellation (Muglach 2008; Liu et al. 2011; Young \& Muglach 2014a, 2014b). Jets tend to occur repeatedly (Chifor et al. 2008; Chen et al. 2015; Liu et al. 2016), with a period ranging from tens of minutes to hours. It is normally thought that repeated jets occur at the same location with almost the same configuration (Guo et al. 2013; Schmieder et al. 2013).

In this paper, for the first time, we show that at high resolution a jet can have two different phases involving reconnection of different magnetic regions, and we propose a model for such a jet.

\section{Instruments}

The observations used in this paper are taken by the Atmospheric Imaging Assembly (AIA; Lemen et al. 2012) and Helioseismic and Magnetic Imager (HMI; Scherrer et al. 2012) on board the $S D O$ satellite. Full-disk EUV images supplied by the AIA instrument have a cadence of $12 \mathrm{~s}$ and a spatial resolution of 0 "' 6 . Full solar disk line-of-sight (LOS) magnetograms in the photosphere obtained by HMI have a cadence of 45 $\mathrm{s}$ and a spatial resolution of 0.15 . The aia_prep.pro is used to process AIA level 1.5 data and HMI LOS data. After the reduction of this routine, the data of AIA and HMI will have the same spatial resolution and the full-disk images will be coaligned. It is not safe to reach conclusions about alignment to within $1^{\prime \prime}-2^{\prime \prime}$ purely from the default SSW software alone because of thermal effects that can cause changes in alignment between two different telescopes (Orange et al. 2014). However, in the present study we align HMI and AIA 1600 images over a broader field of view (about 100" $\times 100^{\prime \prime}$ ), in order to include the region around the sunspot. This plage does indeed align well with the default SSW alignment, and so we are confident about our AIA 171 alignment. 

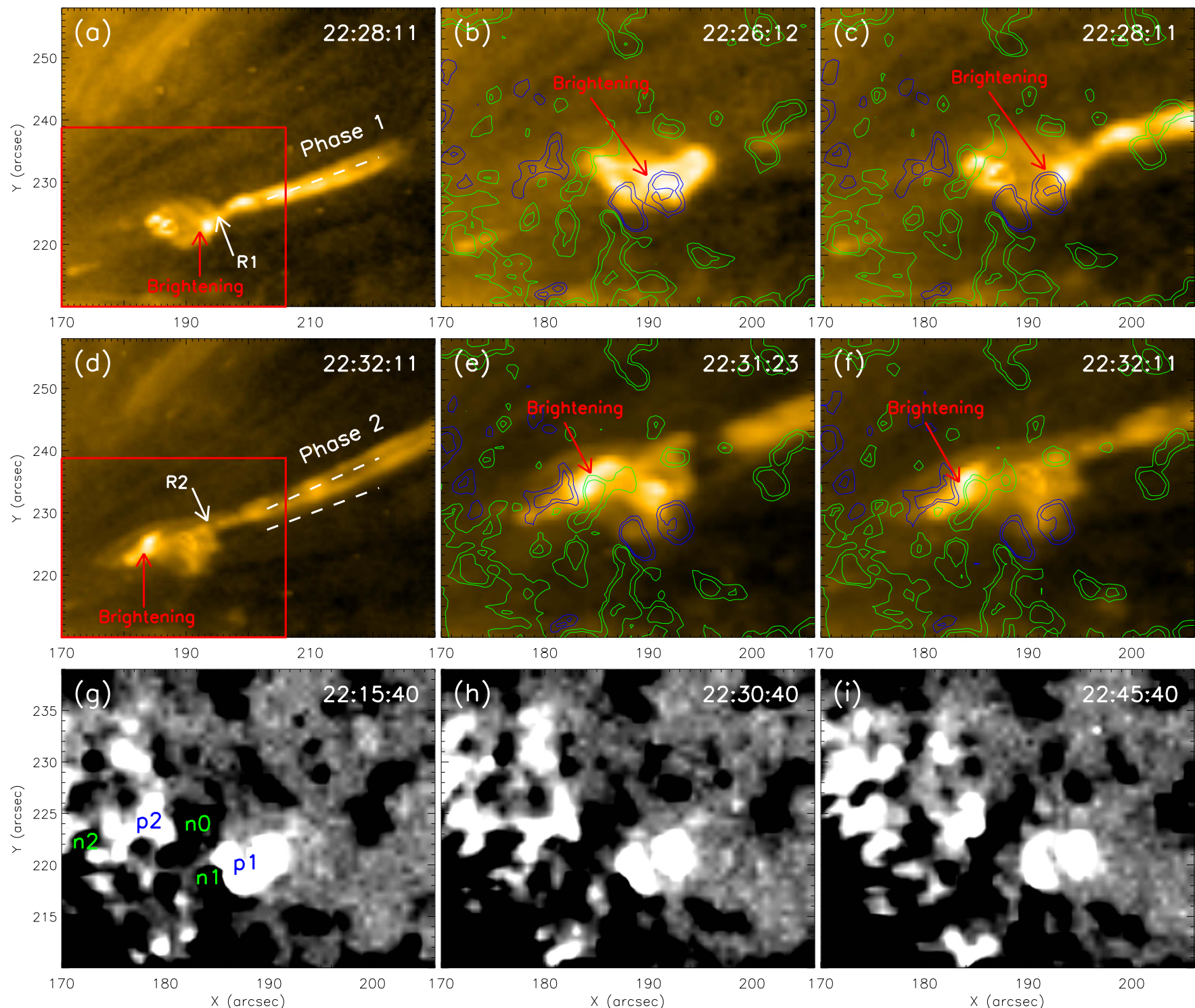

Figure 1. (a) Image of Phase 1 of the jet obtained from SDO/AIA $171 \AA$ at 22:28:11 UT on 2012 July 2 . The red box is the field of view (FOV) for panels (b) and (c). (b)-(c) Close-up SDO/AIA $171 \AA$ images near the base of Phase 1 at 22:26:12 UT and 22:28:11 UT overlaid with simultaneous LOS magnetic field. Blue/green lines mark contour levels for the positive/negative polarities. (d) Image of Phase 2 obtained from SDO/AIA $171 \AA$ at 22:32:11 UT. The red box marks the FOV of panels (e) and (f). (e)-(f) Close-up images near the base of the jet in Phase 2 at 22:31:23 UT and 22:32:11 UT overlaid with simultaneous LOS magnetic field. (g)-(i) SDO/ HMI LOS magnetograms at 22:15 UT, 22:30 UT, and 22:45 UT, separately. The dynamic range for the magnetic field strength is 30 G. The white/black color represents positive/negative polarity. The FOV is the same as the FOV in panels (b), (c), (e), and (f). The negative-polarity region in the middle of the FOV is marked by $\mathrm{n} 0$, and positive-polarity regions to both sides of $\mathrm{n} 0$ are marked by $\mathrm{p} 1$ and $\mathrm{p} 2$, separately. $\mathrm{n} 1$ and $\mathrm{n} 2$ are also marked in panel (g).

\section{Observations and Results}

A series of jets were observed on the west edge of AR 11513 on 2012 July 2 (Chen et al. 2015). A jet with two phases occurring around 22:30 UT is studied. The peak of Phase 1 was around 22:28 UT. Phase 2 followed Phase 1 quickly, and the peak was around 22:32 UT. The configurations of Phase 1 and Phase 2 are shown in Figures 1(a) and (d), with the Phase 2 spire being almost parallel to the Phase 1 spire. However, the images demonstrate clearly that the roots of Phase 1 and Phase 2 are located in different places. The root of the Phase 1 spire originates from the southwest of the jet base, which is marked by R1, while the root of the Phase 2 spire originates from the northeast of the jet base, which is marked by R2. The distance between R1 and R2 is around $2.8 \mathrm{Mm}$. The two spires also appear in different locations: the spire of Phase 2 is to the northeast of the Phase 1 spire by about $2.8 \mathrm{Mm}$.

The evolution process from Phase 1 to Phase 2 is shown in the online animated Figure 2. Originally, there is a bright patch at the jet base that is located between the negative magnetic fragment "n0" and the positive fragment "p1" (Figures 1(b) and $(\mathrm{g}))$. Then the spire of Phase 1 becomes longer and longer and also has a lateral motion to the northeast. Around 22:28:11 UT, we can see clearly the jet spire (Figure 1(c)), and the bright patch becomes smaller and weaker. Around 22:29:23 UT, the root of the jet varies from southwest to northeast. From this time, Phase 1 changes to Phase 2, and during Phase 2, the jet continually moves to the northeast laterally. At 22:31:23 UT, there is a bright patch between "n0" and "p2" to the northeast of the jet base (Figures 1(e) and (g)). At 22:32:11 UT, the 


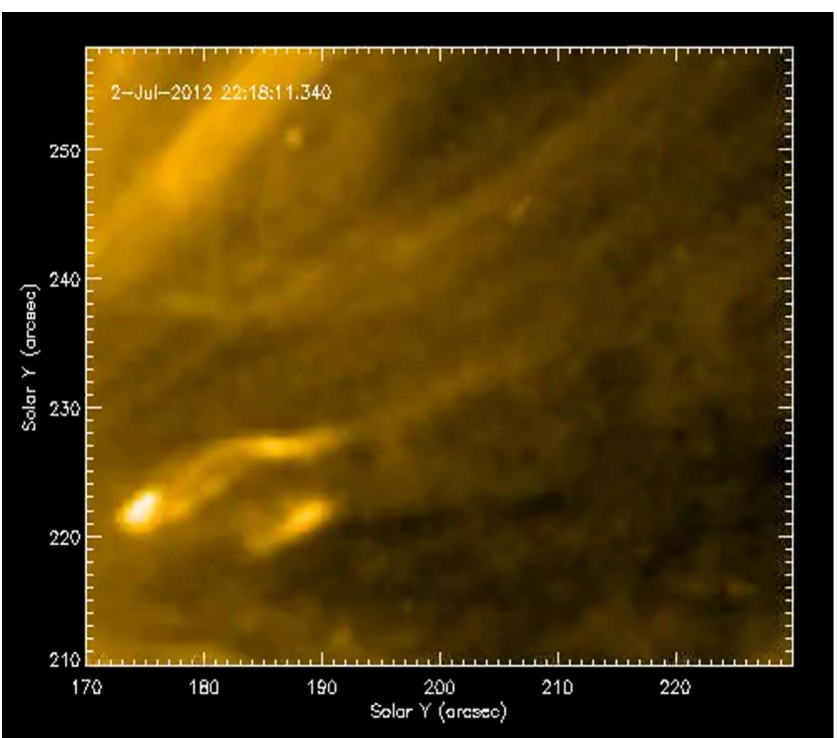

Figure 2. Animation of the $S D O /$ AIA $171 \AA$ data from 22:18:11 to $22: 38: 11$ UT on 2012 July 2. The animation is $3.4 \mathrm{~s}$ in duration.

(An animation of this figure is available.)

whole spire of Phase 2 can be seen with its root located in the northeast (Figures 1(d) and (f)).

At the jet base, there are mixed magnetic polarity regions. In the middle, there is a negative magnetic polarity region that is marked by "n0" in Figure 1(g). To the right of "n0," there is a positive magnetic polarity region marked by "p1"; to the left of "n0," there is another positive magnetic polarity region marked by "p2." n0 and p1 had existed for a long time, but during the occurrence of the jet $\mathrm{p} 1$ and $\mathrm{n} 1$ decreased, while $\mathrm{p} 2$ and $\mathrm{n} 2$ flowed out from the penumbra and then moved toward n0. The evolution of the LOS magnetograms is shown in the online animated Figure 3. The magnetic fragments $\mathrm{n} 0, \mathrm{p} 1$, and $\mathrm{p} 2$ are moving magnetic features. Below the jet the magnetic field is continually changing. From HMI LOS magnetograms in Figures 1(g)-(i), we can see the way the regions n0, p1, and p2 are reducing. Combining images from AIA and HMI, we find that Phase 1 originates from a region between the negativepolarity region $\mathrm{n} 0$ and the positive-polarity region $\mathrm{p} 1$, while Phase 2 originates from the region between $\mathrm{n} 0$ and $\mathrm{p} 2$.

In Figure 1, the jet and the bright patches of the footpoints of the two phases appear in AIA $171 \AA$ A. Actually, the jet and the bright patches are seen in six wavelengths: $171 \AA, 193 \AA$, $211 \AA, 335 \AA, 94 \AA$, and $131 \AA$. The configurations of the two phases in AIA $131 \AA$ are shown in Figure 4; their configurations are similar to that in $171 \AA$. The normalized intensity variations of the bright patch (red box in the image $131 \AA$; Figure 4(a)) in Phase 1 in six wavelengths are shown in Figure 4(a). From 22:24 UT to 22:30 UT, there are three obvious peaks in the six wavelengths. This implies that the bright patch becomes brighter three times during the growth of Phase 1, and the bright patch has a wide temperature range, as shown in the six wavelengths of AIA. The normalized intensity variation of the Phase 2 bright patch (red box in the image $131 \AA$ A; Figure 4(b)) in six wavelengths is shown in Figure 4(b). From 22:30 UT to 22:34 UT, the trends of intensity variation are the same, and there is an obvious peak in the different

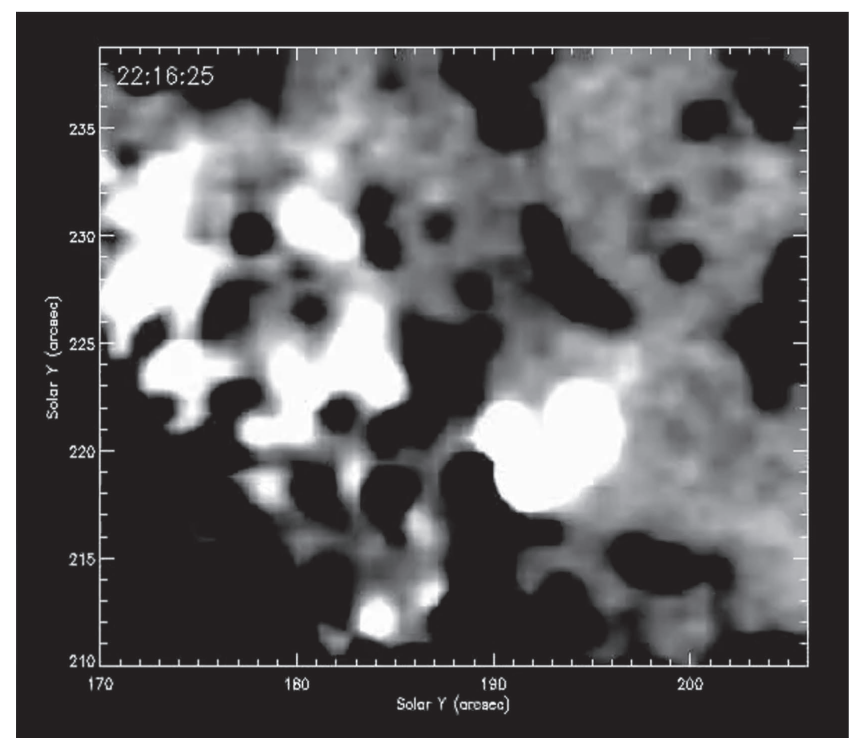

Figure 3. Animation of the $S D O / \mathrm{HMI}$ LOS magnetograms from 22:16:25 to 22:45:40 UT on 2012 July 2 . The animation is $1.4 \mathrm{~s}$ in duration.

(An animation of this figure is available.)

wavelengths. The peak time of this bright patch is the same as Phase 2 of the jet.

In order to know whether there is magnetic cancellation or not, the variation of magnetic flux is calculated. Figure 5 shows the variation of the magnetic flux in the rectangular regions including p1 and p2. From Figure 5(a), we can see that the magnetic flux of $\mathrm{p} 1$ decreases from 22:15 UT to 22:24 UT. At 22:26 UT, there is an obvious brightening at the base of Jet 1 . Magnetic cancellation between regions n 0 and $\mathrm{p} 1$ appears to drive Phase 1. The net increase of magnetic flux of $\mathrm{p} 1$ from 22:24 UT to 22:45 UT, despite the continued cancellation, is due to two small positive-polarity patches (highlighted with two green circles in Figure 5(b)) moving into the p1 region. The variation of magnetic flux in region $\mathrm{p} 2$ is shown in Figure 4(b): it decreases from 22:15 to 22:30 UT. At 22:31 UT, there is a brightening at the base of the jet, suggesting that flux cancellation between regions $\mathrm{n} 0$ and $\mathrm{p} 2$ drives Phase 2 .

The motions and magnetic evolution of $\mathrm{p} 1$ and $\mathrm{p} 2$ are shown in Figure 6. We take a slit between $\mathrm{n} 0$ and p1 (Figure 6(a)) and plot a time-distance image (Figure 6(c)). Figure 6(c) shows that there is magnetic cancellation between $\mathrm{p} 1$ and $\mathrm{n} 0$. The magnetic flux of p1 decreases from 22:00 UT to 23:00 UT. Another slit between p2 and $\mathrm{n} 0$ is also taken, which is marked by a green line in Figure 6(b). The time-distance image is given in Figure 6(d) and shows that $\mathrm{p} 2$ moves toward $\mathrm{n} 0$ from 22:00 UT to 22:30 UT.

\section{Interpretation and Discussion}

The formation and evolution of two successive phases of a jet have been observed here at high spatial resolution. The two phases overlap in time, with a time between their peaks of only 4 minutes. The distance between the peaks of Phase 1 and Phase 2 is only $2.8 \mathrm{Mm}$. The two patches $\mathrm{p} 1$ and $\mathrm{p} 2$ are very close; if the spatial resolution were low, they would not easily be separated from each other. At high resolution we find that Phases 1 and 2 have different configurations: the root of Phase 1 is in the southwest, whereas the root of Phase 2 is in the 

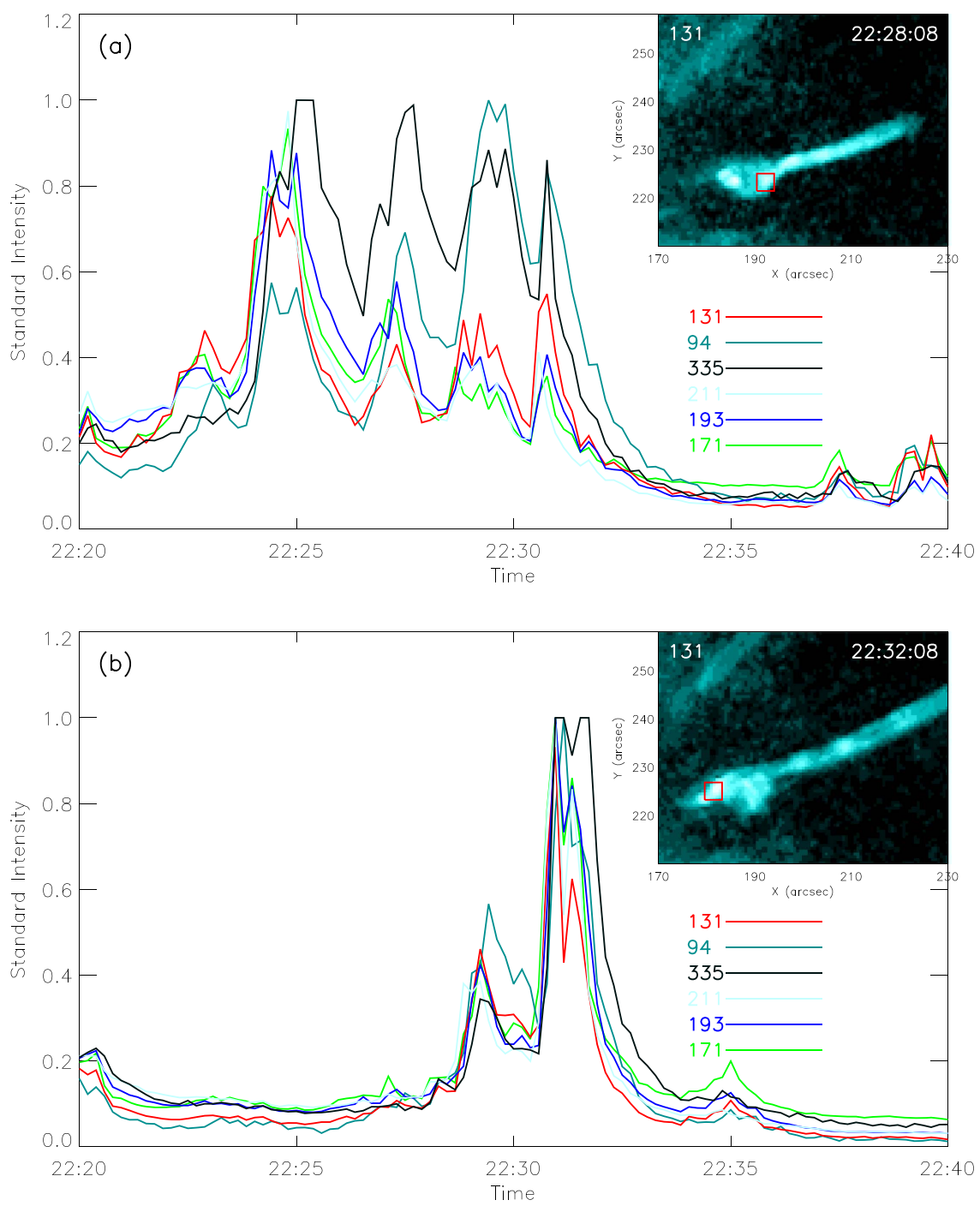

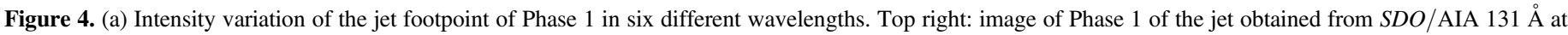

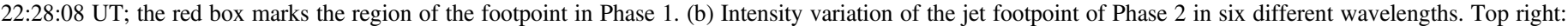
image of Phase 2 of the jet obtained from SDO/AIA $131 \AA$ at 22:32:08 UT; the red box marks the region of the footpoint in Phase 2 .

northeast. The locations of brightening of the feet of the two phases are different: the location of the brightening of the footpoint of Phase 1 is between $\mathrm{p} 1$ and $\mathrm{n} 0$, whereas the location of brightening of the footpoint of Phase 2 is between $\mathrm{p} 2$ and $\mathrm{n} 0$. The two phases and the brightening of their footpoints appear over a wide temperature range. We suggest that the two phases are driven by magnetic flux cancellation but originate from different nearby locations, rather than originating from the same place.

The jets occurred to the west of NOAA AR 11513 in a moat region surrounding a sunspot of negative polarity and containing mixed polarity moving magnetic features. Thus, the ambient magnetic field is predominantly open and directed away from the sunspot. Within this field, small positive magnetic fragments such as $\mathrm{p} 1$ and $\mathrm{p} 2$ tend to form closed flux that is connected to nearby negative fragments. As they move around and come closer to other negative fragments, cancellation and reconnection naturally occur to form new connections, jets and heating. Thus, when different positive fragments cancel with the same negative flux, they tend to produce jets with successive phases that have similar paths due to the nature of the ambient field. There is also a big coronal hole to the west edge of NOAA AR 11513 with open negative magnetic field (Figure 1 of Chen et al. 2015).

Based on our observation and analysis, we interpret the physical mechanism for the two successive phases with a twodimensional sketch (Figure 7). Figure 7(a) depicts the magnetic configuration before the jet. It is dominated by an open magnetic field with negative polarity, which is rooted partly in region $\mathrm{n} 0$ in our observation and partly in $\mathrm{n} 1$ and $\mathrm{n} 2$. To the right of $\mathrm{n} 0$, there is a closed magnetic field, with its positive footpoint $\mathrm{p} 1$ linked to part of another nearby negative fragment. On the left side, there is another closed magnetic structure symmetrically placed with its positive footpoint $\mathrm{p} 2$ linking to part of another nearby negative fragment. During the first process of magnetic cancellation (Figures 7(b) and (c)), the footpoint motion of $\mathrm{p} 1$ and $\mathrm{n} 0$ drives magnetic reconnection. Such magnetic reconnection between open and closed magnetic field is called interchange magnetic reconnection (Moore et al. 2013). The result of the reconnection is to create new closed magnetic flux between $\mathrm{n} 0$ and $\mathrm{p} 1$ and new open magnetic flux in the faraway negative-polarity region $\mathrm{n} 1$. The hot plasma 

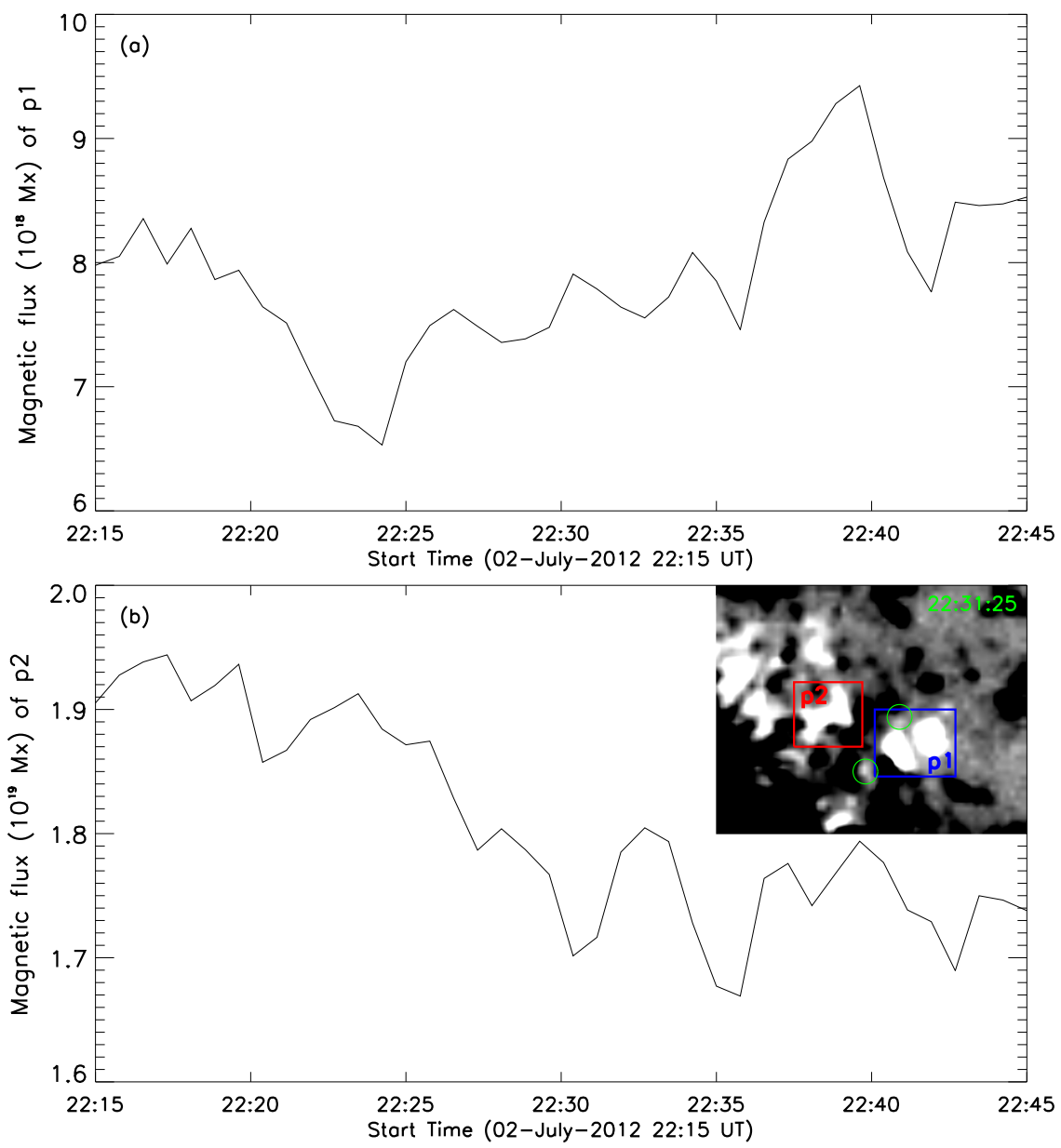

Figure 5. Variation of magnetic flux in regions (a) p1 and (b) p2 from 22:15 UT to 22:45 UT on 2012 July 2. Top right: HMI LOS magnetogram at 22:31 UT; FOV: $36^{\prime \prime} \times 28^{\prime \prime} 8$.

trapped in the new closed-field region creates a bright patch between $\mathrm{n} 0$ and $\mathrm{p} 1$, and hot plasma escapes out along the new open field to become the spire of Phase 1. The spire is collimated by the dominant strong open magnetic flux. Heat from the reconnection is magnetically confined to a small volume in the closed field below the reconnection location and so shows up as a brightening, but in the open field above the reconnection site it is spread over a much larger volume and so does not lead to a brightening in region $\mathrm{n} 1$. The continuous magnetic cancellation makes the jet move sideways. It can be seen, by comparing Figures 7(b) and (c), that the location of the open field line that has just reconnected (and therefore the spire) moves to the left as reconnection proceeds. This transverse motion can be faster than the photospheric cancellation speed, since the transverse motion of photospheric sources can lead to a buildup of a current sheet that subsequently reconnects. Phase 2 is driven by the subsequent cancellation between $\mathrm{n} 0$ and $\mathrm{p} 2$ (Figure 7(d)) and produces a spire and a brightening in the same manner as in Phase 1. It is possible that the two phases are independent of one another, but this is unlikely since they occur so close in time and space and the spire has a more or less smooth motion, which suggests that the first phase helped trigger the second phase or that the fragments $\mathrm{p} 1$ and $\mathrm{p} 2$ are not independent but are linked somehow by a common convection pattern in the moat below the photosphere.
The formation and evolution process of the jet is a process in which magnetic energy is converted to kinetic energy and thermal energy via magnetic reconnection. From our observations, we estimate the magnetic energy, kinetic energy, and thermal energy. For Phase 1 during magnetic cancellation from 22:15 UT to 22:24 UT, the magnetic flux decreases by $1.5( \pm 0.2) \times 10^{18} \mathrm{Mx}$ in about 9 minutes (Figure 5(a)), resulting in a mean magnetic cancellation rate of $(2.8 \pm 0.3) \times 10^{15} \mathrm{Mx}$ $\mathrm{s}^{-1}$. The length of region $\mathrm{p} 1$ is around $6.0 \times 10^{8} \mathrm{~cm}$. We calculate the mean magnetic energy release rate during the time of magnetic cancellation to be $(2.8 \pm 0.1) \times 10^{27} \mathrm{erg} \mathrm{s}^{-1}$, which is similar to the change of magnetic energy in a reconnection event described by $\mathrm{Li}$ et al. (2016). Following the calculation of the plasma density and temperature by Liu et al. (2016), the average electron number density is about $10^{9} \mathrm{~cm}^{-3}$, and the mean temperature is $1.5 \mathrm{MK}$ for the jet. For the bright patch at the footpoint of the jet, the density is about $2.5 \times 10^{9} \mathrm{~cm}^{-3}$ and the temperature is $4 \mathrm{MK}$. For the jet, the velocity is about $200 \mathrm{~km} \mathrm{~s}^{-1}$, the width is about $4 \mathrm{Mm}$, the length is about $30 \mathrm{Mm}$, and we estimate the mass of the jet to be $3.7 \times 10^{11} \mathrm{~g}$. The change of kinetic energy is $7.5 \times 10^{25} \mathrm{erg} \mathrm{s}^{-1}$. Likewise, we estimate the change of thermal energy of the jet spire to be $7.8 \times 10^{25} \mathrm{erg} \mathrm{s}^{-1}$. For the bright patch at the footpoint of the jet, the length is about $8 \times 10^{8} \mathrm{~cm}$ and the thermal energy is $7.1 \times 10^{26} \mathrm{erg} \mathrm{s}^{-1}$. About $30 \%$ of the magnetic energy is converted to kinetic and thermal energy, so the 

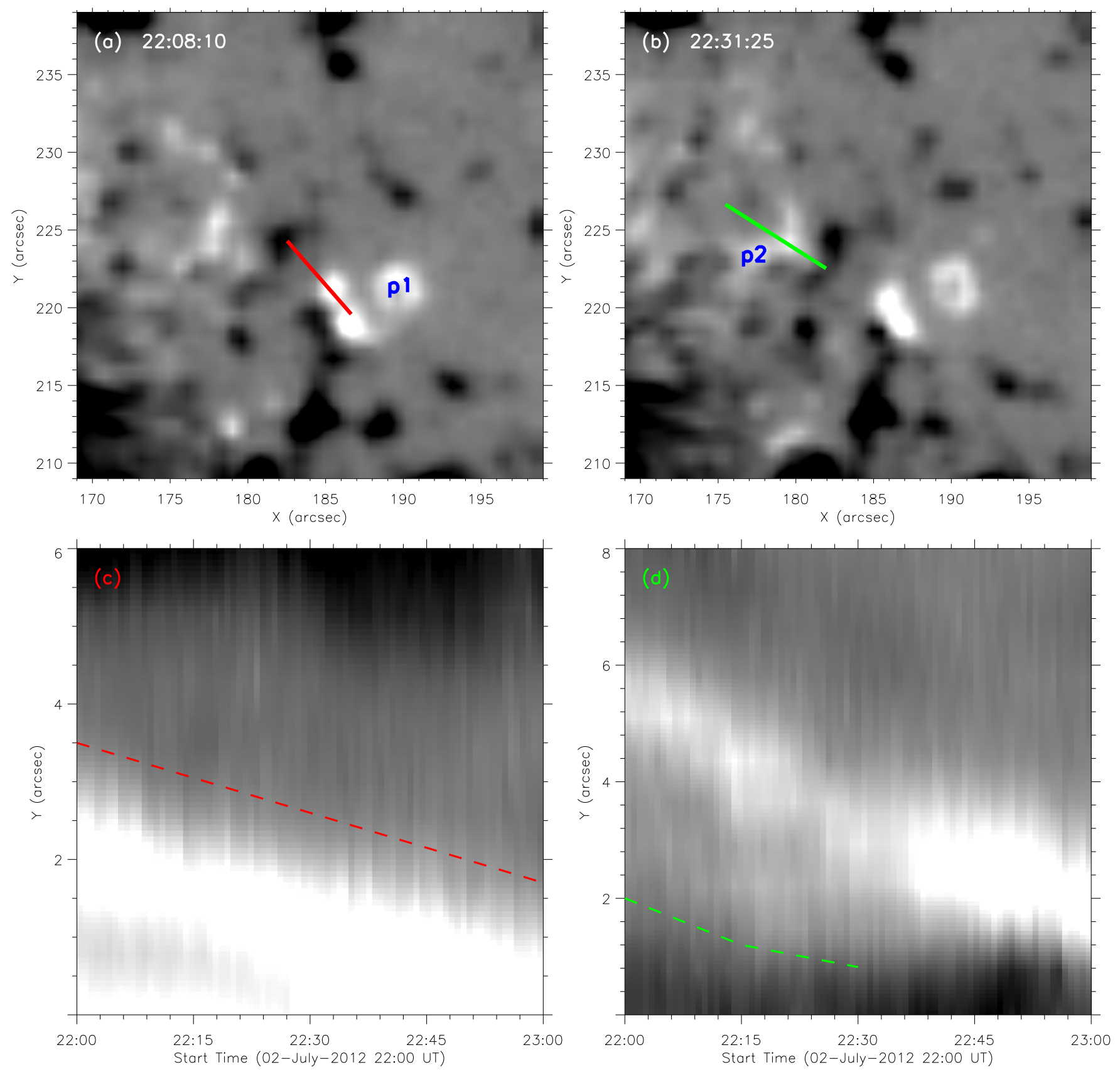

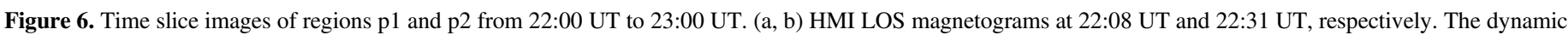

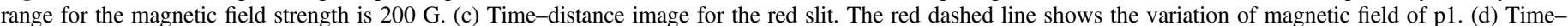
distance image for the green slit. The green dashed line shows the variation of magnetic field of p2.

magnetic energy is sufficient to provide the thermal energy and kinetic energy of the jet (more details of the calculation can be found in the Appendix).

We are thankful for discussion with Dr. Qingmin Zhang. $S D O$ is a mission for NASA's Living With a Star program. This work was partly supported by National Natural Science Foundation of China (grant Nos. 11303048, 11673033, $11373040,11427901)$. This work was also partly supported by an International Exchanges cost share award with NSFC for overseas travel between collaborators in the UK and China, and State Key Laboratory for Space Weather, Center for Space Science and Applied Research, Chinese Academy of Sciences.

\section{Appendix \\ Energy Estimation}

Here we describe the estimates of magnetic energy, kinetic energy, and thermal energy for Phase 1 of the jet.

The magnetic flux $\Phi$ decreases by $1.5( \pm 0.2) \times 10^{18} \mathrm{Mx}$ in about 9 minutes from 22:15 UT to 22:24 UT (Figure 5(a)). The magnetic flux density $\mathrm{B}$ can be expressed as

$$
B=\frac{\Phi}{S}
$$

where $\Phi$ is the variation of magnetic flux and $S$ is the area of magnetic cancellation. The length of the region $\mathrm{p} 1(L)$ is around 

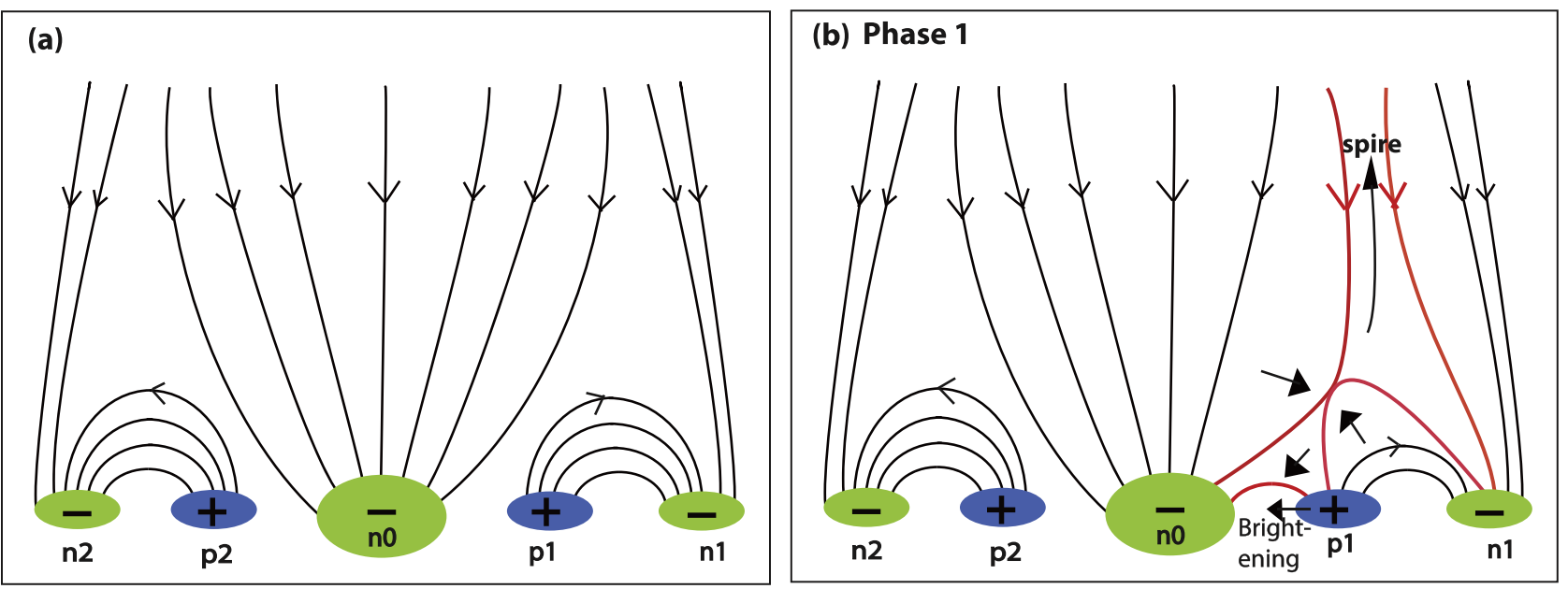

(c) Phase 1-lateral motion of spire

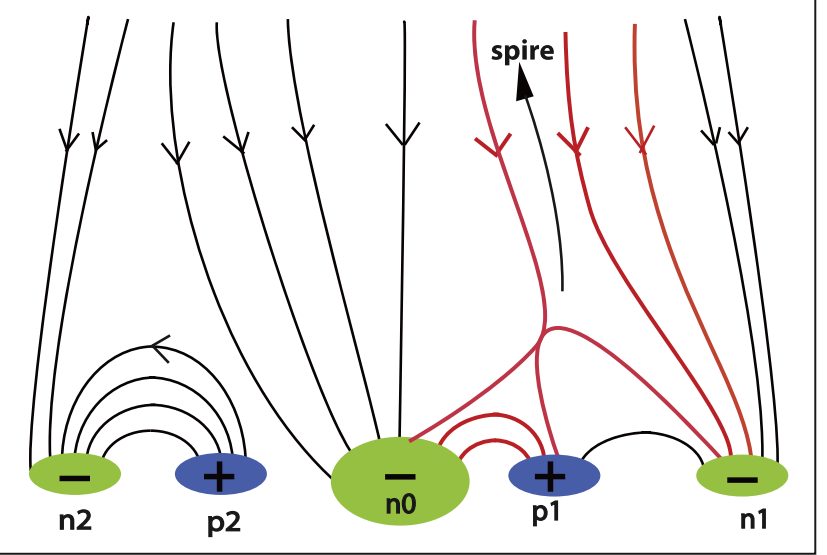

(d) Phase 2

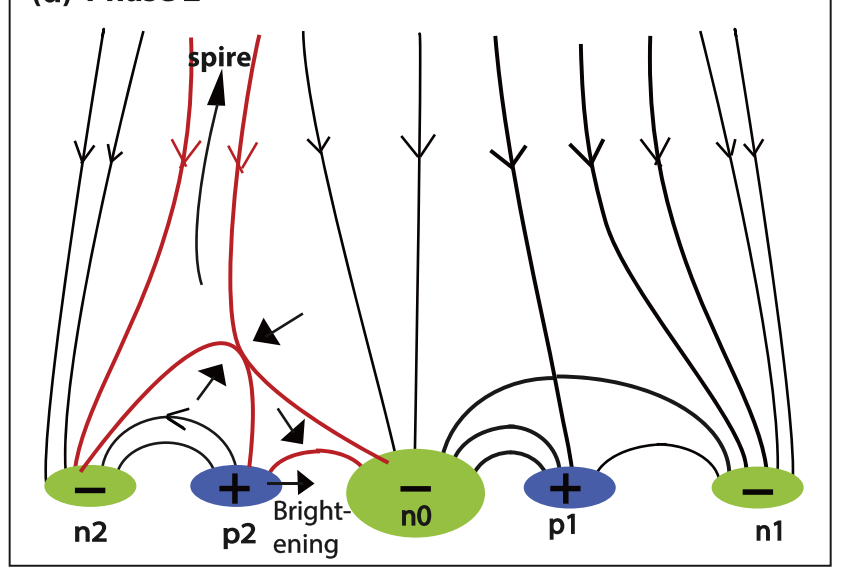

Figure 7. Schematic of the topology and magnetic reconnection in the two successive phases of a complex jet showing a few representative field lines marked by lightheaded arrows before and after bursts of reconnections. (a) Before reconnection $\mathrm{n} 0$ and the outer parts of $\mathrm{n} 1$ and $\mathrm{n} 2$ are open magnetic fields with negative polarity. On both sides of $\mathrm{n} 0$, there are closed magnetic fields with positive polarities $\mathrm{p} 1$ and $\mathrm{p} 2$ linking to the inner parts of the negative polarities $\mathrm{n} 1$ and $\mathrm{n} 2$, as shown. Arrows mark the direction of magnetic field lines. (b) Phase 1 of the reconnection process driven by magnetic cancellation between $\mathrm{n} 0$ and $\mathrm{p} 1$. Reconnection takes place at the X-point, with plasma velocities shown by solid-headed arrows. The upward-moving outflow jet from the reconnection site is channeled upward by the open field lines as a spire. The separatrices and the field lines that have reconnected (a new open field line and a new closed field line) are marked in red. The location of a chromospheric brightening is also indicated below the reconnection location and straddling $\mathrm{n} 0$ and $\mathrm{p} 1$. (c) Phase 1 continues with more reconnection at the $\mathrm{X}$-point and so creates a lateral motion of the spire. (d) Phase 2 repeats the same process, but at the X-point on the left side of n0, driven by magnetic cancellation as $\mathrm{p} 2$ approaches n0. The separatrices and the newly reconnected field lines are shown in red.

$6.0 \times 10^{8} \mathrm{~cm}$, and we assume that the width is $1 \%$ of the length (namely, $6.0 \times 10^{6} \mathrm{~cm}$ ). The variation of magnetic flux density $B$ is around $420 \mathrm{G}$ during the period of magnetic cancellation.

The mean magnetic energy release rate $E_{m}$ during the time of magnetic cancellation $(t)$ can be written as

$$
E_{m}=\frac{B^{2}}{8 \pi \times t} V \text {. }
$$

The length of the region $\mathrm{p} 1$ is around $6.0 \times 10^{8} \mathrm{~cm}(L)$, so the volume $V$ is $L^{3}$ and $E_{m}$ is $(2.8 \pm 0.1) \times 10^{27} \mathrm{erg} \mathrm{s}^{-1}$.

The change of kinetic energy is

$$
E_{k}=\frac{1}{2} m v^{2}
$$

where $m$ is the mass of the accelerated plasma in Phase 1 . Here $m$ is set to be $\mu m_{0} n_{e} \pi L(d / 2)^{2}$, where $\mu$ is the mean molecular weight 0.58 for fully ionized coronal plasma, $m_{0}$ is the mass of protons, the width $d$ is about $4 \times 10^{8} \mathrm{~cm}$, the length $L$ is about $3 \times 10^{9} \mathrm{~cm}$, and $n_{e}$ is the estimated average electron number density of Phase 1 . The plasma density $n_{e}$ for Phase 1 is about $10^{9} \mathrm{~cm}^{-3}$, and the velocity $v$ is about $200 \mathrm{~km} \mathrm{~s}^{-1}$. Using these parameters, we obtain the mass of accelerated plasma in Phase 1 as $3.7 \times 10^{11} \mathrm{~g}$ and the change of kinetic energy as $7.5 \times 10^{25} \mathrm{erg} \mathrm{s}^{-1}$.

The thermal energy of the jet is

$$
E_{\mathrm{th}}=N \kappa_{p} T,
$$

where $\kappa_{p}$ is the Boltzmann constant, $N=n_{e} V$ is the number of electrons involved in Phase 1 , and $V$ is the volume. $T$ is the average temperature, which we take to be $1.5 \mathrm{Mk}$. The thermal energy of the jet spire is $7.8 \times 10^{25} \mathrm{erg} \mathrm{s}^{-1}$. For the bright patch at the footpoint of the jet, the density is about $2.5 \times 10^{9} \mathrm{~cm}^{-3}$, the temperature is $4 \mathrm{Mk}$, and the length is about $8 \times 10^{8} \mathrm{~cm}$, so the thermal energy is $7.1 \times 10^{26} \mathrm{erg}$. 
The mean magnetic energy release rate is $2.8 \times 10^{27} \mathrm{erg} \mathrm{s}^{-1}$, while the sum of the kinetic energy and thermal energy of the jet spire is $1.5 \times 10^{26} \mathrm{erg}$, and the thermal energy of the bright patch at the footpoint is $7.1 \times 10^{26} \mathrm{erg}$. Thus, about $30 \%$ of the magnetic energy is converted to kinetic and thermal energy.

Note that for Phase 1 of the jet, the average electron number density is $10^{9} \mathrm{~cm}^{-3}$ and the mean temperature is $1.5 \mathrm{MK}$. For the bright patch at the footpoint of the jet, density $2.5 \times 10^{9} \mathrm{~cm}^{-3}$ and temperature $4 \mathrm{MK}$ are taken from Liu et al. (2016).

\section{References}

Archontis, V., \& Hood, A. W. 2013, ApJ, 769, 21

Brooks, D. H., Kurokawa, H., \& Berger, T. E. 2007, ApJ, 656, 1197

Canfield, R. C., Peardon, K. P., Leka, K. D., et al. 1996, ApJ, 464, 1016

Chae, J., Qiu, J., Wang, H., \& Goode, P. R. 1999, ApJL, 513, L75

Chen, J., Su, J., Yin, Z., et al. 2015, ApJ, 815, 71

Chifor, C., Young, P. R., Isobe, H., et al. 2008, A\&A, 481, L57

Cirtain, J. W., Golub, L., Lundquist, L., et al. 2007, Sci, 318, 1580

Forbes, T. G., \& Priest, E. R. 1984, SoPh, 94, 315

Guo, Y., Dmoulin, P., Schmieder, B., et al. 2013, A\&A, 555, A19

Heyvaerts, J., Priest, E. R., \& Rust, D. M. 1977, ApJ, 216, 123

Hong, J., Jiang, Y., Yang, J., et al. 2016, ApJ, 830, 60

Jiang, R. L., Fang, C., \& Chen, P. F. 2012, ApJ, 751, 152

Lemen, J. R., Title, A. M., Akin, D. J., et al. 2012, SoPh, 275, 17

Li, L. P., Zhang, J., Peter, H., et al. 2016, NatPh, 12, 847

Liu, C., Deng, N., Liu, R., et al. 2011, ApJL, 735, L18
Liu, J. J., Wang, Y. M., Erdelyi, R., et al. 2016, ApJ, 833, 150

Liu, J. J., Wang, Y. M., Liu, R., et al. 2014, ApJ, 782, 94

Moore, R. L., Cirtain, J. W., Sterling, A. C., \& Falconer, D. A. 2010, ApJ, 720,757

Moore, R. L., Sterling, A. C., Falconer, D. A., \& Robe, D. 2013, ApJ, 769, 134 Moreno-Insertis, F., \& Galsgaard, K. 2013, ApJ, 771, 20

Muglach, L. 2008, ApJ, 687, 1398

Nishizuka, N., Shimizu, M., Nakamura, T., et al. 2008, ApJ, 683, 83

Nóbrega-Siverio, D., Moreno-Insertis, F., \& Martínez-Sykora, J. 2016, ApJ, 822,18

Orange, N. B., Oluseyi, H. M., Chesny, D. L., et al. 2014, SoPh, 289, 1901

Raouafi, N. E., Patsourakos, S., Pariat, E., et al. 2016, SSRv, 201, 1

Scherrer, P. H., Schou, J., Bush, R. I., et al. 2012, SoPh, 275, 207

Schmieder, B., Guo, Y., Moreno-Insertis, F., et al. 2013, A\&A, 559, A1

Shen, Y., Liu, Y., Su, J., \& Deng, Y. , 2012, ApJ, 745, 164

Shibata, K., Ishido, Y., Acton, L. W., et al. 1992, PASJ, 44, 173

Shibata, K., Nitta, N., Strong, K. T., et al. 1994, ApJL, 431, L51

Shimojo, M., Hashimoto, S., Shibata, K., et al. 1996, PASJ, 48, 123

Shimojo, M., Shibata, K., \& Harvey, K. L. 1998, SoPh, 178, 379

Sterling, A. C., Moore, R. L., Falconer, D. A., \& Adams, M. 2015, Natur, 523, 437

Syntelis, P., Archontis, V., Gontikakis, C., \& Tsinganos, K. 2015, A\&A, 584,10

Yokoyama, T., \& Shibata, K. 1995, Natur, 375, 42

Yokoyama, T., \& Shibata, K. 1996, PASJ, 48, 353

Young, P. R., \& Muglach, K. 2014a, PASJ, 66, 12

Young, P. R., \& Muglach, K. 2014b, SoPh, 289, 3313

Zhang, Q. M., \& Ji, H. S. 2014, A\&A, 561, A134

Zhang, Q. M., Ji, H. S., \& Su, Y. N. 2016, SoPh, 291, 859 